\title{
Adsorption of Si and C Atoms over SiC (111) Surfaces
}

\author{
P. G. Vivas, E. E. da Silva, L. C. de Carvalho, J. L. A. Alves, H. W. Leite Alves, \\ Departamento de Ciências Naturais - FUNREI, \\ C.P.: 110, CEP: 36300-000, São João del Rei - MG, BRAZIL
}

L. M. R. Scolfaro, and J. R. Leite

LNMS, Departamento de Física dos Materiais e Mecânica, USP, C.P.: 66.318, CEP: 05389-970, São Paulo, SP, Brazil

Received on 23 April, 2001

\begin{abstract}
In this work, using Density Functional, Hartree-Fock and Extended Hückel Theories together with the supercell and cluster model approaches, we present our preliminary results for the simulation of the adsorption of $\mathrm{Si}$ and $\mathrm{C}$ atoms over the (111) SiC surfaces as well as the torsion of $\mathrm{SiC}$ molecules at that surfaces. Our results shown that, before and after the adsorption, the cubic structure are the most stable one, when compared with the hexagonal ones. However, the torsional energy barrier between the cubic and the hexagonal structures is small enough that, at the conditions of $\mathrm{SiC}$ growth, it facilitates the $\mathrm{SiC}$ polytype formation.
\end{abstract}

\section{Introduction}

The recent interest on the physical properties of $\mathrm{SiC}$, is due to the fact that this compound is the most promising substrate for the growth of III-Nitrides. Also, the material is the ideal one for the fabrication of semiconductor devices operating under extreme conditions $[1,2]$. Moreover, $\mathrm{SiC}$ has a large number of polytypes and the knowledge of their properties is a key for both successful growth of the III-Nitrides and the hightemperature microelectronic devices. The polytypes, or stable long-range order modifications, are described as different stacking sequences of $\mathrm{SiC}$ layers along the [111] or the [0001] directions of the corresponding cubic or hexagonal structures [3-6].

Despite the progress achieved on the growth of $\mathrm{SiC}$ by MOCVD(or by MBE), lots of small tricks(such as heating the substrate or creating buffer layers) have been employed to obtain a specific modification of such material $[7,8]$. Moreover, there is no experimental $\mathrm{SiC}$ thermodynamic phase diagram, which it could be a good guide for the knowledge of the mechanisms of the polytype formation.

On the theoretical side, there have been some progress on the understanding the structural, dynamical and electronic properties of $\mathrm{SiC}$ and its polytypes [3-6]. However, much of these efforts are based on the static pictures and equilibrium approaches for the processes occurring in a $\mathrm{MBE}$ (or CVD) transformation [9].
In this case, total energy differences calculations between polytypes have been done, as well as Ising simulations, and, until now, there is not a real consensus about the mechanisms of polytype formation [10].

In this work, we present our preliminary results for the atomic and electronic structures of the $\mathrm{Si}$ and $\mathrm{C}$ atoms adsorbed over both C-terminated and $\mathrm{Si}-$ terminated $\mathrm{SiC}$ (111) surfaces in order to understand the mechanisms of the polytype formation. The obtained results were calculated by means of the conjugation of the Density Functional(DFT), HartreeFock(HF) and Extended Hückel(EHT) Theories together with the supercell and cluster model approaches. The models, separately, have been successfully used to characterize the III-Nitrides surfaces [11] and now, they will be applied together to study Si- and C-terminated $\mathrm{SiC}(111)$ surfaces in a $(1 \times 1)$ reconstruction pattern.

In our cluster model calculations, we have used Unrestricted HF method with single-zeta basis set(STO3G), within the Gaussian94 code [12]. We have then calculated the optimised uppermost atomic positions and the resulting electronic structures of the Si6C4H13, C6Si4H13(simulating, respectively, the Siand C-terminated $\mathrm{SiC}$ (111) surfaces), $\mathrm{Si}($ or $\mathrm{C})+$ Si6C4H13 and $\mathrm{Si}($ or C) + C6Si4H13(simulating the adsorption of the $\mathrm{C}$ and $\mathrm{Si}$ atoms over $\mathrm{SiC}(111)$ surfaces) clusters. In these calculations the inner atoms were kept fixed at their $\mathrm{Si}-\mathrm{C}, \mathrm{Si}-\mathrm{H}$, and $\mathrm{C}-\mathrm{H}$ bond distances, which are, respectively, $1.89 \AA$, 1.54 Åand $1.09 \AA$ A11]. 
The hydrogen atoms were introduced to prevent artificial dangling bonds on the surface.

Now, for our slab supercell calculations, we have used both DFT, in the Local Density Approximation, and the crystalline EHT methods. The DFT calculations were done within the Car-Parrinello approach within the fhi96md code [13], with the TroullierMartins pseudopotentials [14], and the Ceperley-Alder exchange term as parametrized by Perdew and Zunger [15]. In this particular calculation, we use symmetric slabs consisting of four $\mathrm{SiC}$ double layers plus two central Si layers(in the case of Si-terminated surface) or two central C layers(for the C-terminated one), similar to that proposed by Wenzien et al. [16]. The lattice constants were fixed at the bulk theoretical equilibrium values, $a_{0}=4.341 \AA$, converged with 60 Ryd of cutoff energy on the plane-wave expansion. We used vacuum regions with a thickness equivalent to four $\mathrm{SiC}$ double layers. The central layers of the slab were kept fixed at the bulk positions and the outermost layers of atoms on both sides of the slab were relaxed to geometries given by the calculated total energies and forces. The equilibrium geometry is identified when all forces are smaller than $0.01 \mathrm{eV} / \AA$. This corresponds to a numerical uncertainty of atomic positions of less than $0.01 \AA$. The summation over four Monkhorst-Pack special k-points in the irreducible part of the Brillouin-zone was used to replace Brillouin-zone integrations.

The EHT calculations were done within the BiconCedit code, proposed by Calzaferri et al., which have applied sucessfully on calculations of the electronic properties of some Carbon compounds [17]. This particular version of EHT method is based in a new way to express the weighted Wolfsberg-Helmholtz parameter $K$, which is now distance-dependent, having only two parameters: $\kappa$ and $\delta$. In order to obtain these parameters for $\mathrm{SiC}$, we have parametrized the lattice parameter and the Bulk modulus for the cubic modification, using ten Monkhorst-Pack special k-points in the calculations. The obtained values were $\kappa=0.87$ and $\delta=0.56 \AA^{-1}$, which lead to $\mathrm{a}_{0}=4.19 \AA$ And $\mathrm{B}_{0}$ $=2.03 \mathrm{Mbar}$, in good agreement with the experimental results. As a final check, we simulate the electronic structure of the most common polytypes $(2 \mathrm{H}, 4 \mathrm{H}, 3 \mathrm{C}$ and $6 \mathrm{H}$ ), and our results for the total energy differences between the polytypes agree well with other theoretical calculations $[4,5]$. For the slab calculations, we used seven $\mathrm{SiC}$ double layers and, for the vacuum region, five $\mathrm{SiC}$ double layers. The calculation were performed with seven Monkhorst-Pack special k-points.

\section{Results and discussion}

In table I, we show the results for the relaxation of the C- and Si-terminated $\mathrm{SiC}(111)(1 \times 1)$. Here, only the surface atoms, in our cluster model calcultions, were left to relax, while in the slab calculations, the surface and the sub-surface atoms were left to relax. Surprisingly, the results show that these surfaces relaxes with small strains $\Delta z$. These results are consistent with other calculations [16], and with the experimental results [18]. Besides that, our calculations show that the Si-terminated is the most stable one, when compared with the C-terminated in this reconstruction pattern. Considerations about other reconstruction patterns will be published soon in another publication.

To simulate the adsorption of any atom over the (111) surface, we must consider the following adsorption sites [16]: T1, T4 and H3, which are over, respectively, a surface atom, a sub-surface atom and a second sub-layer atom. Our simulations about that for Si and $\mathrm{C}$ atoms adsorbed over C-terminated $\mathrm{SiC}$ surfaces, they prefer the $\mathrm{T} 1$ site, which $\Delta \mathrm{z}=1.85 \AA$ And $1.27 \AA$, respectively. It is interesting to note that, Si try to keep the same interatomic $\mathrm{SiC}$ distance, $1.89 \AA$, while the $\mathrm{C}-\mathrm{C}$ distance is less than the $\mathrm{C}-\mathrm{C}$ interatomic distance in diamond, which is $1.54 \AA$.

Table 1: Summary of the calculated surface strains $\Delta z($ in $\AA)$ for the relaxation of the $3 \mathrm{C}-\mathrm{SiC}(111)(1 \times 1)$ surfaces. $\Delta \mathrm{z}_{1}$ is related to the top surface atoms, and $\Delta \mathrm{z}_{2}$, to the first sub-layer.

\begin{tabular}{lcccc}
\hline \hline & Si-terminated & \multicolumn{3}{c}{ C-terminated } \\
\hline & $\Delta \mathrm{z}_{1}$ & $\Delta \mathrm{z}_{2}$ & $\Delta \mathrm{z}_{1}$ & $\Delta \mathrm{z}_{2}$ \\
$\mathrm{HF}$ & -0.06 & - & -0.01 & - \\
EHT & 0.00 & 0.00 & -0.07 & 0.0 \\
DFT & -0.06 & 0.04 & - & - \\
\hline \hline
\end{tabular}

Our simulations for the $\mathrm{Si}$ and $\mathrm{C}$ adsorption over Si-terminated surfaces show, on the contrary, that the adsorbates prefer the T4 site, in good agreement with other calculations [18]. In this case $\Delta \mathrm{z}=1.37 \AA$ And 0.43 Afor the adsorption of $\mathrm{Si}$ and $\mathrm{C}$, respectively. A more detailed analysis of the electronic states and their connection to the atomic structure of the adsorbed surface is in progress and will come up soon in another publication.

Finally, we also have simulate the torsion of the adsorbed Si over the C-terminated $\mathrm{SiC}(111)$ surface, using the molecular cluster model. To do that, we add more three hydrogen atoms on the adsorbed $\mathrm{Si}$ in order to simulate the two possible configurations for the next adsorbed layer: the "cis" configuration, which leads to hexagonal structures, or the "trans" one, giving cubic 
structures. Our results showed that the cubic modification is, at $0 \mathrm{~K}$, the most stable. However, the energy barrier between the two configurations is around $84 \mathrm{meV}$, which is extremally smaller. We can infer, from this result, that at the temperature conditions of the $\mathrm{SiC}$ growth, we could not distinguish what kind of configuration can the adsorbed layers be, i.e., if it is in a "cis" or in a "trans" configuration. So, this could be the reason for the large number of $\mathrm{SiC}$ polytypes found in the nature.

\section{Final Remarks}

In summary, we have presented our preliminary results for the atomic and electronic structures of the $\mathrm{Si}$ and $\mathrm{C}$ atoms adsorbed over both $\mathrm{C}$-terminated and Siterminated $\mathrm{SiC}$ (111) surfaces in order to understand the mechanisms of the polytype formation. Our results shown that, the relaxations of the $(1 \times 1)$ reconstruction pattern are small, and the Si-terminated surface is more stable than the C-terminated one. We also, simulate the adsorption of $\mathrm{Si}$ and $\mathrm{C}$ atoms over the reconstructed surface. Our results show that, after the adsorption, the T1 and T4 sites are the most stable one for the, respectively, $\mathrm{C}$ - and Si-terminated surfaces. Finally, the torsional energy barrier between the cubic and the hexagonal structures is small enough that, at the conditions of $\mathrm{SiC}$ growth, it facilitates the $\mathrm{SiC}$ polytype formation.

\section{Acknowledgements}

This work was partially supported by $\mathrm{CNP}_{\mathrm{q}}$ and by FAPEMIG.

\section{References}

1. S. C. Jain, M. Willander, J. Narayan, and R. van Overstraeten, J. Appl. Phys. 87, 965 (2000).

2. R. S. Kern and R. F. Davies, Mater. Sci. Eng. B46, 240 (1997).
3. G. L. Vignoles, J. Cryst. Growth 118, 430 (1992).

4. P. Käckell, B. Wenzien, and F. Bechstedt, Phys. Rev. B50, 17037 (1994).

5. S. Limpijumnong and W. R. L. Lambrecht, Phys. Rev. B57, 12017 (1998).

6. C. -Z. Wang, R. Yu, and H. Krakauer, Phys. Rev. BB53, 5430 (1996); F. Widulle, T. Ruf, O. Buresch, A. Debernardi, and M. Cardona, Phys. Rev. Lett. 82, 3089 (1999).

7. R. S. Kern, S. Tanaka, L. B. Rowland, and R. F. Davies, J. Cryst. Growth 183, 581 (1998).

8. C. Bittencourt, Semicond. Sci. Technol. 15, 1115 (2000).

9. J. Y. Tsao, Materials Fundamentals of Molecular Beam Epitaxy (Academic Press, San Diego, 1993).

10. V. Heine, C. Cheng, and R. J. Needs, Mater. Sci. Eng. B11, 55 (1992).

11. J. L. A. Alves, H. W. Leite Alves, C. de Oliveira, R. D. S. C. Valadão, and J. R. Leite, Mater. Sci. Eng. B43, 288 (1997); Mater. Sci. Eng. B 50, 57 (1997); H. W. Leite Alves, J. L. A. Alves, J. L. F. da Silva, J. R. Leite and R. A. Nogueira, Mater. Sci Eng. B59, 258(1999); H. W. Leite Alves, J. L. A. Alves, R. A. Nogueira, and J. R. Leite, Braz. J. Phys. 29, 817 (1999).

12. Gaussian 94, Revision D.4, M. J. Frisch et al., Gaussian Inc., Pittsburgh PA, 1995.

13. M. Bockstedte, A. Kley, J. Neugebauer, and M. Scheffler, Comput. Phys. Comm. 107, 187 (1997).

14. N. Troullier and J. L. Martins, Phys. Rev. B43, 1993 (1991).

15. J. P. Perdew and A. Zunger, Phys. Rev. B23, 5048 (1981).

16. B. Wenzien, P. Käckell and F. Bechstedt, Surf. Sci. 331-333, 1105 (1995).

17. G. Calzaferri and R. Rytz, J. Phys. Chem. 100, 11122(1996); G. Calzaferri, L. Forss, and I. Kamber, J. Phys. Chem. 93, 5366 (1989).

18. R. Kaplan, Surf. Sci. 215, 111 (1989). 